\title{
Non-invasive imaging through opaque scattering layers
}

\author{
J. Bertolotti ${ }^{a}$, E.G. van Putten ${ }^{b}$, C. Blum ${ }^{c}$, A. Lagendijk ${ }^{b}$, W.L. $\operatorname{Vos}^{b}$, A.P. Mosk ${ }^{b}$ \\ ${ }^{a}$ Physics and Astronomy Department, University of Exeter, UK; \\ ${ }^{b}$ Complex Photonic Systems (COPS), University of Twente, NL; \\ ${ }^{c}$ Nanobiophysics, University of Twente, Enschede, NL
}

\begin{abstract}
Light scattering is known for blurring images to the point of making them appear as a white halo. For this reason imaging through thick clouds or deep into biological tissues is difficult. Here we discuss in details a method we developed recently to retrieve the shape of an object hidden behind a diffusing screen.
\end{abstract}

\section{INTRODUCTION}

In order to form an image our eyes rely on the rules of ray optics. The crystalline lens map light coming from different directions to different points on the retina, and together with the knowledge of the lens' accommodation allows to obtain a reliable picture of our surrounding. But when the light coming at us is scattered before reaching our eyes, as it might happen when we are immersed in a deep fog, this directional information is lost and the quality of the picture is degraded. Up to the point when we can't discern what surround us anymore. This is especially a problem in the field of biophotonics, where tissues strongly scatter light and thus non-invasive imaging is mostly limited to a shallow depth below the surface. ${ }^{1}$

Due to the huge importance of this problem, in the last decades many different approaches were proposed and demonstrated, each with its advantages and disadvantages. For semi-transparent media it is possible to perform imaging using gated techniques to separate the small amount of ballistic light that did not change direction from the scattered background..$^{2-5}$ Yet the achievable resolution rapidly degrades when the scattering becomes stronger ${ }^{6}$ and this method breaks down when all ballistic light is blocked. Alternatively diffuse wave tomography techniques can locate absorptive objects deep inside a scattering medium ${ }^{7}$ but do not permit to resolve details. ${ }^{8}{ }^{9}$ It has been theoretically suggested that a complete knowledge of the scattering screen will allow one to image objects hidden behind it. ${ }^{10}$ Major steps in this direction were achieved using ultrasound ${ }^{11}$ and electromagnetic waves in both the microwave ${ }^{12,13}$ and in the optical regime. ${ }^{14-21}$ Yet to obtain the required knowledge of the scattering screen, it is necessary to access its back, thus severely limiting the usefulness of these approach.

We recently described a method to retrieve the shape of a fluorescent object hidden behind a thin but strongly scattering, and thus completely opaque, screen. ${ }^{22}$ Here we discuss in details, with the help of synthetic data, the working principle of the method, its potential, limitations and how to avoid some of the possible pitfalls. This also forms the basis to understand and exploit several papers that replicated and extended our work. ${ }^{23-25}$

\section{THE METHOD}

The geometry we will consider is depicted in Figure 1. A fluorescent object is placed behind a scattering slab while a coherent light beam is shone at an angle $\boldsymbol{\theta}$ from the front. When coherent light is elastically scattered it produces speckle, ${ }^{26}$ so the hidden object will be illuminated by an irregular, and unknown, pattern of bright and dark spots. If we call $s(\mathbf{x}, \boldsymbol{\theta})$ the intensity speckle pattern generated by a beam at an angle $\boldsymbol{\theta}$ that illuminate the object and $o(\mathbf{x})$ the position-dependent density of fluorophores in the hidden object, than the total amount of fluorescence produced will be proportional to the superposition of the speckle with the fluorescent object. If

Send correspondence to j.bertolotti@exeter.ac.uk

Adaptive Optics and Wavefront Control for Biological Systems, edited by Thomas G. Bifano,

Joel Kubby, Sylvain Gigan, Proc. of SPIE Vol. 9335, 93350W · @ 2015 SPIE

CCC code: $1605-7422 / 15 / \$ 18 \cdot$ doi: $10.1117 / 12.2079525$

Proc. of SPIE Vol. $933593350 \mathrm{~W}-1$ 


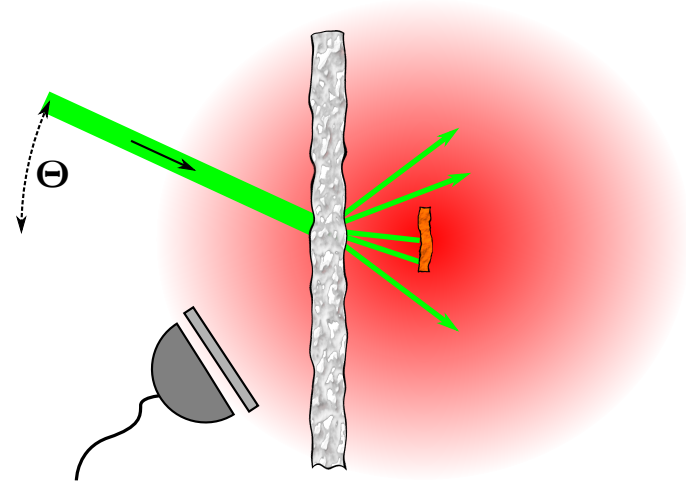

Figure 1. Schematic depiction of the experimental system. A fluorescent object is positioned on the right of the scattering screen. On the left we have a coherent light beam that illuminates the screen at a variable angle $\boldsymbol{\theta}$ and a detector with a filter to cut the illumination and collect only the fluorescence from the hidden object.

the scattering slab and the detector are static, the fraction of fluorescence that we can detected will be constant. So we can write the collected amount of light as

$$
I(\boldsymbol{\theta})=\alpha \int o(\mathbf{x}) s(\mathbf{x}, \boldsymbol{\theta}) d^{2} \mathbf{x}
$$

where $\alpha$ is the collection efficiency.

\subsection{The optical memory effect}

Speckle might appear to be random, but it isn't completely so. In particular speckle is known to be characterized by the presence of several correlations ${ }^{27,28}$ that allow to predict, in certain circumstances, how the intensities at two different points are related. The correlation we are interested in here is known as the optical memory effect, and describes how the speckle generated by a coherent beam impinging on a scattering layer is related to the speckle generated by a beam that impinges on the same scattering layer but with a different angle of incidence. ${ }^{27,29}$ Due to the fact that the disordered system that the light has to traverse is the same, it turns out that if we tilt by a small angle $\boldsymbol{\Delta} \boldsymbol{\theta}$ the beam, the speckle pattern will not change, but just rotate by the same angle and change gradually while the tilt angle becomes bigger.

Calling $\bar{s}(\boldsymbol{\theta})=\int s(\mathbf{x}, \boldsymbol{\theta}) d^{2} \mathbf{x}$ the average intensity of a speckle pattern and $\delta s(\mathbf{x}, \boldsymbol{\theta})=s(\mathbf{x}, \boldsymbol{\theta})-\bar{s}(\boldsymbol{\theta})$ the oscillations around this mean, we can describe how much the speckle pattern changes using the correlation function $^{28}$

$$
\mathcal{C}(\boldsymbol{\Delta} \mathbf{x}, \boldsymbol{\Delta} \boldsymbol{\theta})=\left\langle\frac{\iint \delta s(\mathbf{x}, \boldsymbol{\theta}) \delta s(\mathbf{x}+\boldsymbol{\Delta} \mathbf{x}, \boldsymbol{\theta}+\boldsymbol{\Delta} \boldsymbol{\theta}) d^{2} \mathbf{x} d^{2} \boldsymbol{\theta}}{\bar{s}^{2}(\boldsymbol{\theta})}\right\rangle=\frac{\langle\delta s \star \delta s\rangle}{\bar{s}^{2}},
$$

where $\star$ represents the cross-correlation product $(f \star g)(\Delta x)=\int f^{*}(x) g(x+\Delta x) d x$ and the angular brackets represent an ensemble average over all realization of speckle. Considering only the dominant term (under the assumptions that we are far away from Anderson localization ${ }^{30,31}$ ) we find ${ }^{27,28}$

$$
\mathcal{C}(\boldsymbol{\Delta} \mathbf{x}, \boldsymbol{\Delta} \boldsymbol{\theta})=e^{-k^{2}(\boldsymbol{\Delta} \mathbf{x}-d \boldsymbol{\Delta} \boldsymbol{\theta})^{2} \sigma^{2}} \cdot\left(\frac{k|\boldsymbol{\Delta} \boldsymbol{\theta}| L}{\sinh (k|\boldsymbol{\Delta} \boldsymbol{\theta}| L)}\right)^{2},
$$

where $\sigma$ is the standard deviation of the gaussian incident beam, $d$ is the distance of the object from the screen, $L$ is the layer thickness and $k$ is the wave vector. The first term represents the autocorrelation of the speckle pattern for a fixed angle of incidence, and rapidly approach a Dirac Delta centered at $\boldsymbol{\Delta} \mathbf{x}=d \boldsymbol{\Delta} \boldsymbol{\theta}$ when the width of the incident beam increases. The second term represent how much the speckle pattern changes when changing $\boldsymbol{\theta}$ and decay to half of the maximum value at $\boldsymbol{\Delta} \boldsymbol{\theta} \simeq 1.5 /(k L)$. Interestingly eq. 3 is completely independent from how scattering the layer is. As a consequence the applicability of the method we describe is not determined by 

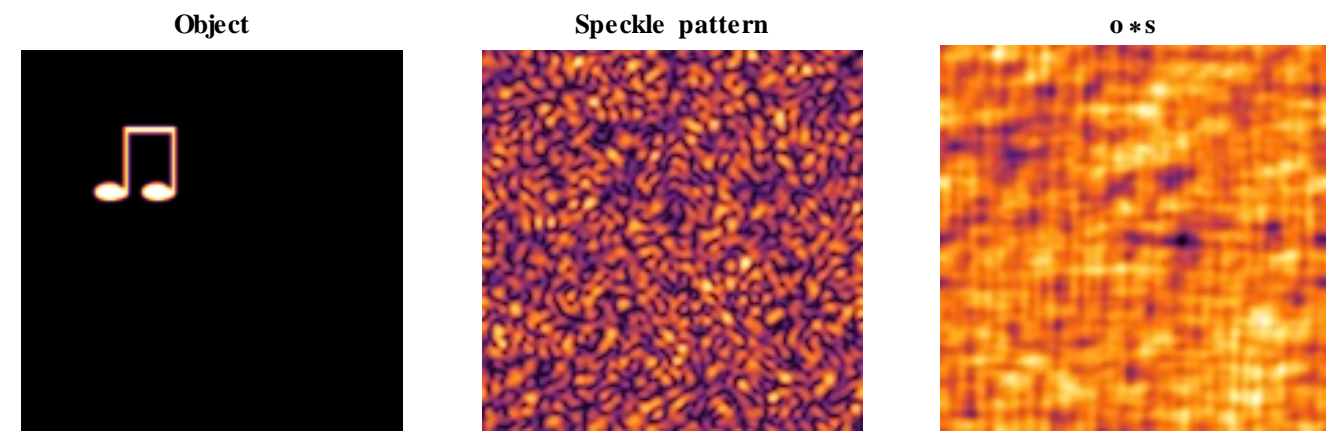

Figure 2. Synthetic data showing (from left to right) a pattern representing the hidden object, a speckle pattern and their convolution.

the optical density of the medium, as it is common with many other methods ${ }^{6}$ making it particularly useful when thin but strongly scattering layers are present.

\subsection{Measuring autocorrelations}

The optical memory effect can be exploited to shift the unknown speckle pattern over the hidden fluorescent object in a controlled way. If the optical memory range is large, i.e. the speckle pattern does not change appreciably when we rotate the incident beam by $\boldsymbol{\Delta} \boldsymbol{\theta}$, eq. 1 takes the convenient form

$$
I(\boldsymbol{\theta})=\alpha \int o(\mathbf{x}) s(\mathbf{x}-d \boldsymbol{\theta}) d^{2} \mathbf{x}=\alpha[o * s](\boldsymbol{\theta}),
$$

i.e. a $2 \mathrm{D}$ function of the incidence angle that is proportional to the convolution between $o$ and $s$. As shown in Figure 2 the randomness of the speckle result in a measured $[o * s](\boldsymbol{\theta})$ that bears very little resemblance with the original object, although general features like the presence of vertical and horizontal lines can be seen upon a closer inspection. We can measure $I(\boldsymbol{\theta})$ directly by sequentially collecting the fluorescence for many angle of incidence but, in order to extract informations about the function $o$, i.e. the shape of the hidden object, we need to autocorrelate $I$ :

$$
\begin{aligned}
{[I \star I](\boldsymbol{\Delta} \boldsymbol{\theta}) } & =\alpha^{2} \int\left(\int o(\mathbf{x}) s(\mathbf{x}, \boldsymbol{\theta}) d^{2} \mathbf{x}\right)\left(\int o(\mathbf{y}) s(\mathbf{y}, \boldsymbol{\theta}+\boldsymbol{\Delta} \boldsymbol{\theta}) d^{2} \mathbf{y}\right) d^{2} \boldsymbol{\theta}= \\
& =\alpha^{2} \iint o(\mathbf{x}) o(\mathbf{y})\left(\int s(\mathbf{x}, \boldsymbol{\theta}) s(\mathbf{y}, \boldsymbol{\theta}+\boldsymbol{\Delta} \boldsymbol{\theta}) d^{2} \boldsymbol{\theta}\right) d^{2} \mathbf{x} d^{2} \mathbf{y}= \\
& =\alpha^{2} \iint o(\mathbf{x}) o(\mathbf{y})((s \star s)(|\mathbf{x}-\mathbf{y}|, \boldsymbol{\Delta} \boldsymbol{\theta})) d^{2} \mathbf{x} d^{2} \mathbf{y} .
\end{aligned}
$$

To go further we have to notice that the autocorrelation of $s$ and the autocorrelation of $\delta s$ differ just by a constant:

$$
(\delta s \star \delta s)=((s-\bar{s}) \star(s-\bar{s}))=(s \star s)+(\bar{s} \star \bar{s})-2(s \star \bar{s})=(s \star s)-(\bar{s} \star \bar{s})=(s \star s)-A \bar{s}^{2},
$$

where $A$ is the area covered by the speckle we are autocorrelating. Making an ensamble average $\langle\cdot\rangle$, performing the change of variable $\Delta \mathbf{x}=\mathbf{y}-\mathbf{x}$ and using eq. 3 and 6 in eq. 5 we obtain

$$
\begin{aligned}
\langle I \star I\rangle & \boldsymbol{\Delta} \boldsymbol{\theta})=\alpha^{2} \int\left(\int o(\mathbf{x}) o(\mathbf{x}+\boldsymbol{\Delta} \mathbf{x}) d^{2} \mathbf{x}\right)\left(\langle\delta s \star \delta s\rangle(\boldsymbol{\Delta} \mathbf{x}, \boldsymbol{\Delta} \boldsymbol{\theta})+A \bar{s}^{2}\right) d^{2} \boldsymbol{\Delta} \mathbf{x}= \\
& =\alpha^{2} \int\left(\int o(\mathbf{x}) o(\mathbf{x}+\boldsymbol{\Delta} \mathbf{x}) d^{2} \mathbf{x}\right)\left(\bar{s}^{2} \mathcal{C}(\boldsymbol{\Delta} \mathbf{x}, \boldsymbol{\Delta} \boldsymbol{\theta})+A \bar{s}^{2}\right) d^{2} \boldsymbol{\Delta} \mathbf{x}= \\
& =\alpha^{2} \bar{s}^{2} \int[o \star o](\boldsymbol{\Delta} \mathbf{x}) e^{-k^{2}(\boldsymbol{\Delta} \mathbf{x}-d \boldsymbol{\Delta} \boldsymbol{\theta})^{2} \sigma^{2}} \cdot\left(\frac{k|\boldsymbol{\Delta} \boldsymbol{\theta}| L}{\sinh (k|\boldsymbol{\Delta} \boldsymbol{\theta}| L)}\right)^{2} d^{2} \boldsymbol{\Delta} \mathbf{x}+\alpha^{2} A \bar{s}^{4} \int[o \star o](\boldsymbol{\Delta} \mathbf{x}) d^{2} \boldsymbol{\Delta} \mathbf{x}= \\
& =\alpha^{2} \bar{s}^{2} \cdot\left(\frac{k|\boldsymbol{\Delta} \boldsymbol{\theta}| L}{\sinh (k|\boldsymbol{\Delta} \boldsymbol{\theta}| L)}\right)^{2} \int[o \star o](\boldsymbol{\Delta} \mathbf{x}) e^{-k^{2}(\boldsymbol{\Delta} \mathbf{x}-d \boldsymbol{\Delta} \boldsymbol{\theta})^{2} \sigma^{2}} d^{2} \boldsymbol{\Delta} \mathbf{x}+\alpha^{2} A \bar{s}^{4}\|o\|^{2}
\end{aligned}
$$



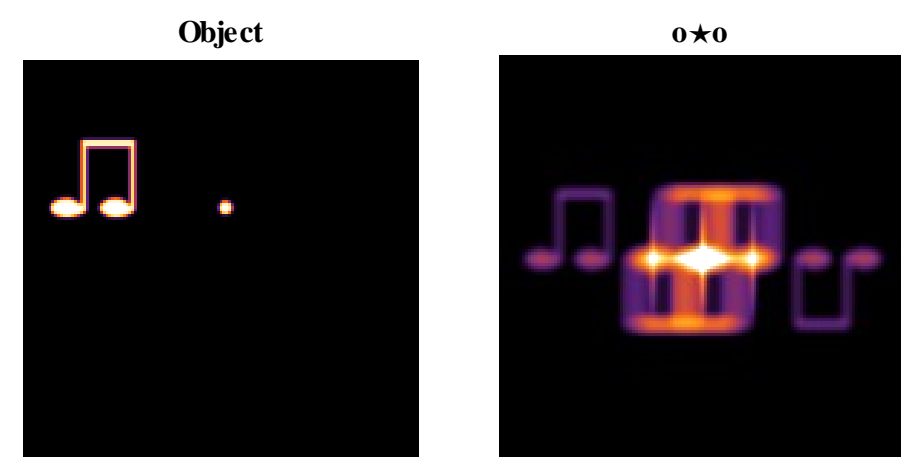

Figure 3. If the function $o(\mathbf{x})$ is composed by the object we are interested in retrieving plus a clearly separated dot, then its autocorrelation contains two clear copies of the object itself.

where $\|o\|^{2}$ is the square of the total integral of $o(\mathbf{x})$.

Apart from the proportionality constant $\alpha^{2} \bar{s}^{2}$, the first term in eq. 7 represents the speckle decorrelation for large $\boldsymbol{\Delta} \boldsymbol{\theta}$ and the second the convolution of the object autocorrelation $(o \star o)$ with a narrow gaussian. Finally the last term is an offset. Therefore, as long as the size of the object is smaller than $2 d \boldsymbol{\Delta} \boldsymbol{\theta}$ the autocorrelation of the measured $I$ is proportional to the autocorrelation of the hidden object $o$ plus a constant.

\subsection{Inverting the autocorrelation}

Even if we are able to measure perfectly $o \star o$, we still need to extract $o$ from it. Since an autocorrelation is a lossy operation, not everything can be retrieved, even in principle. In fact the autocorrelation of any function is always centered at $\boldsymbol{\Delta} \boldsymbol{\theta}=0$, so any information about the absolute position of $o$ is lost, and only relative positions can be found. Furthermore the autocorrelation of a positive and real function must be centrosymmetric, thus creating an ambiguity about the object original orientation.

A particularly simple case occurs when, as shown if Figure 3, the function $o(\mathbf{x})$ is composed by the object we are interested in retrieving plus a clearly separated dot. In this case the autocorrelation $o \star o$ factor in a central part that contains the object autocorrelation and two copies of the object itself on the sides. If we are not that lucky it is important to recover $o \star o$ as accurately as possible. Form eq. 7 we see that, assuming that the object we want to recover is smaller than $d$ times the memory range and that the speckle pattern is fine enough (i.e. $\sigma$ is large), then $o \star o$ is well approximated by $\langle I \star I\rangle$ minus a constant. This poses a conceptual problem, since we have no direct way to estimate $\alpha^{2} A \bar{s}^{4}\|o\|^{2}$ and subtract it. On the other hand we can assume the minimum value of $o \star o$ to be zero ( $o$ is a positive function and thus its autocorrelation must be positive too), and thus subtract any offset from $\langle I \star I\rangle$. This approximated approach proved effective in experimental conditions. ${ }^{22,24}$ It is also important to notice that, in order to properly approximate $o \star o$, we need to perform an ensemble average over the realization of the speckle patterns on $I \star I$. This can be easily obtained by measuring $I(\boldsymbol{\theta})$ for several central angle separated by more than the memory range, so that they correspond each to a different speckle pattern.

The shape of the hidden object can be obtained using a Gerchberg-Saxton-like algorithm ${ }^{32,33}$ that iteratively imposes the fact that the autocorrelation of the object is $o \star o$ and that $o(\mathbf{x})>0$. There are several possible variants of this algorithm, as the ones used in stellar speckle interferometry ${ }^{34}$ or in x-ray scattering. ${ }^{35,36}$ Here we will show a simple implementation in the Mathematica language of the so-called Error Reduction algorithm: ${ }^{33}$ g=RandomReal $[\{0,1\},\{\operatorname{dim}, \operatorname{dim}\}]$;

$\mathrm{mf}=$ Sqrt $[$ Abs [Fourier [oo] ] ]

er [g] := ( $\mathrm{g} 1=\operatorname{Re}[$ InverseFourier[ $\mathrm{mf} * \operatorname{Exp}[\mathrm{I}$ Arg[Fourier[g]]] ] ;

$\mathrm{g} 1=((\mathrm{g} 1+\mathrm{Abs}[\mathrm{g} 1]) / 2) ; \operatorname{Return}[\mathrm{g} 1] ; \mathrm{g} 1=$.$) ;$

$\mathrm{ER}\left[i_{-}\right]:=(\mathrm{g}=\operatorname{Nest}[\mathrm{er}, \mathrm{g}, \mathrm{i}])$;

where $o \circ$ is the variable containing the measured $o \star o$ and dim is its size. A measure of how well the algorithm converged after $i$ iterations of ER can be obtained by calculating $e=|g \star g-o \star o|$. It is important to notice that the error reduction algorithm is known to get stuck into a local minimum from time to time, so a good strategy 

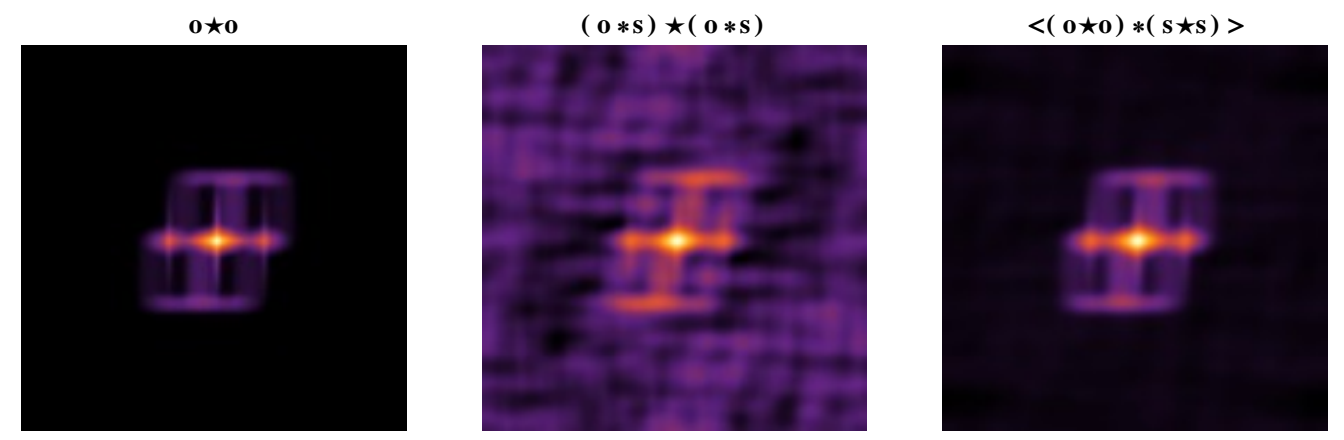

Figure 4. Left panel: autocorrelation of the object. Central panel: the autocorrelation of $o * s$ provides a crude approximation of $o \star o$. Right panel: to remove the noise we can perform an average over the realizations of $s$. The result is a good approximation of $o \star o$ with a slight loss of resolution due to the finite size of the speckle grains, as expected from eq. 7 .

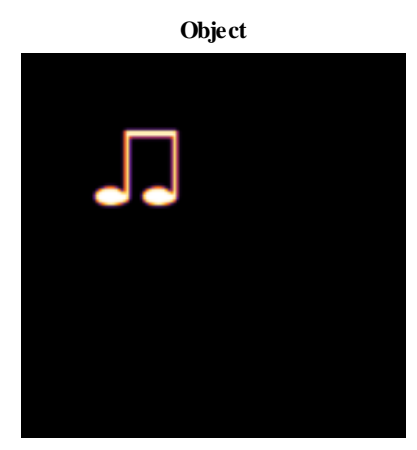

$\mathrm{e}=\mathbf{2 8 2 . 1 7 6}$

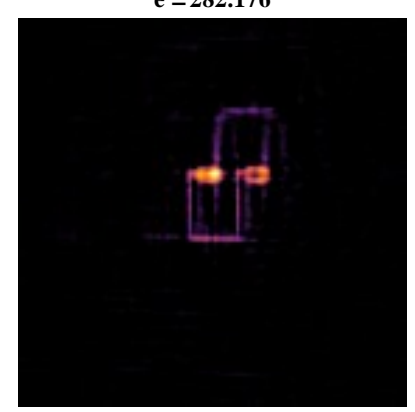

$\mathrm{e}=\mathbf{2 . 9 7 6 4 2}$

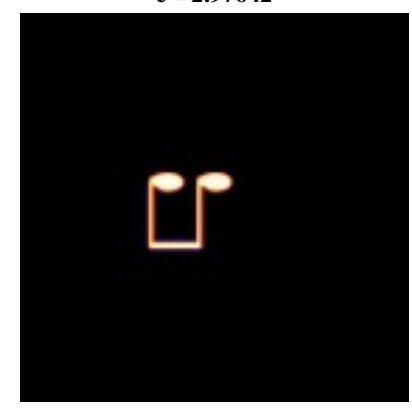

$\mathrm{e}=\mathbf{2 4 8 . 0 9 8}$

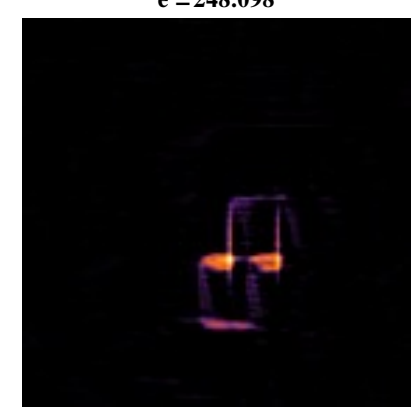

$\mathrm{e}=\mathbf{1 8 0 . 1 5 2}$

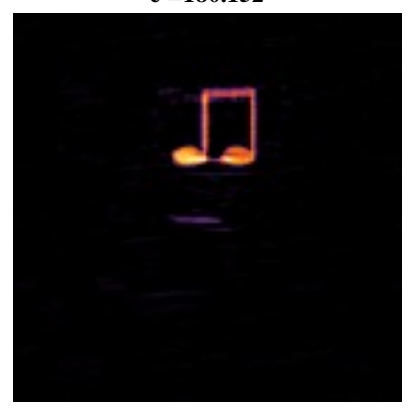

$\mathrm{e}=\mathbf{1 2 4 0 . 2 6}$

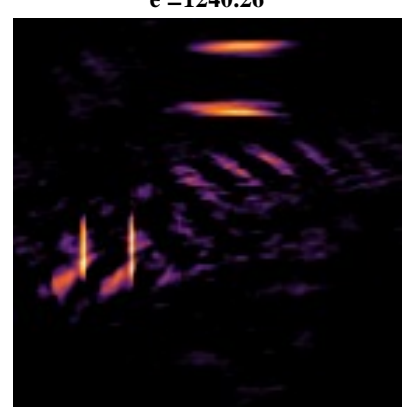

Figure 5. Original object (upper left corner) together with the result of 5 runs of 2000 iterations of the error reduction algorithm.

is to run the algorithm several times and choose the solution that gives the smallest value of $e .^{24}$ In Figure 5 we show the original object and 5 instances of 2000 iterations of the error reduction algorithm, with the associated residual error $e$, for different random initial conditions. As we can see the quality of the reconstruction varies widely, but the value of $e$ is a very good indicator of which reconstruction is the best. We also notice that the various reconstructions do not have a fixed position and that sometimes they appear flipped. This is due, as discussed above, to the ambiguities in the autocorrelation. ${ }^{33}$

\section{CONCLUSIONS}

In this paper we discussed how it is possible to exploit the optical memory effect to measure the autocorrelation of a object hidden behind a scattering layer, and how to retrieve the shape of the object from this autocorrelation. Since our original paper on imaging through a scattering layer ${ }^{22}$ several variations on our method were proposed and demonstrated, ${ }^{23-25}$ but all ultimately rely on the relationship between the measured quantity $\langle I \star I\rangle$ with 
the desired quantity $o \star o$. The main result of this paper is eq. 7, that formally describes this relationship with the only assumption that the scattering layer is diffusive.

\section{REFERENCES}

[1] Ntziachristos, V., "Going deeper than microscopy: the optical imaging frontier in biology," Nat. Methods 7, $603(2010)$.

[2] Abramson, N., "Light-in-flight recording by holography," Opt. Lett. 3, 121-123 (Oct 1978).

[3] Huang, D., Swanson, E. A., Lin, C. P., Schuman, J. S., Stinson, W. G., Chang, W., Hee, M. R., Flotte, T., Gregory, K., Puliafito, C. A., and Fujimoto, J. G., "Optical coherence tomography," Science 254, 1178-1180 (1991).

[4] Nasr, M. B., Saleh, B. E. A., Sergienko, A. V., and Teich, M. C., "Demonstration of dispersion-canceled quantum-optical coherence tomography," Phys. Rev. Lett. 91, 083601 (Aug 2003).

[5] Mujumdar, S. and Ramachandran, H., "Imaging through turbid media using polarization modulation: dependence on scattering anisotropy," Opt. Commun. 241(1-3), 1 - 9 (2004).

[6] Ishimaru, A., Sermsak, J., and Kuga, Y., "Imaging through random multiple scattering media using integration of propagation and array signal processing," Waves in Random and Complex Media 22, 24-39 (2012).

[7] den Outer, P., Nieuwenhuizen, T., and Lagendijk, A., "Location of objects in multiple-scattering media," J. Opt. Soc. Am. A 10, 1209-1218 (JUN 1993).

[8] Yodh, A. and Chance, B., "Spectroscopy and imaging with diffusing light," Phys. Today 48, 34 (1995).

[9] Gibson, A., Hebden, J., and Arridge, S., "Recent advances in diffuse optical imaging," Phys. Med. Biol. 50(4), R1-R43 (2005).

[10] Freund, I., "Looking through walls and around corners," Physica A 168, 49-65 (1990).

[11] Fink, M., "Time reversed accoustics," Phys. Today 50, 34-40 (1997).

[12] Lerosey, G., de Rosny, J., Tourin, A., Derode, A., Montaldo, G., and Fink, M., "Time reversal of electromagnetic waves," Phys. Rev. Lett. 92, 193904 (May 2004).

[13] Lerosey, G., de Rosny, J., Tourin, A., and Fink, M., "Focusing beyond the diffraction limit with far-field time reversal," Science 315(23), 1120-1122 (2007).

[14] Vellekoop, I. M. and Mosk, A. P., "Focusing coherent light through opaque strongly scattering media," Opt. Lett. 32(16), 2309-2311 (2007).

[15] Yaqoob, Z., Psaltis, D., Feld, M. S., and Yang, C., "Optical phase conjugation for turbidity suppression in biological samples," Nat. Photon. 2, 110-115 (2008).

[16] Popoff, S. M., Lerosey, G., Carminati, R., Fink, M., Boccara, A. C., and Gigan, S., "Measuring the transmission matrix in optics: An approach to the study and control of light propagation in disordered media," Phys. Rev. Lett. 104, 100601 (Mar 2010).

[17] Popoff, S., Lerosey, G., Fink, M., Boccara, A. C., and Gigan, S., "Image transmission through an opaque material," Nat. Commun. 1, 81 (2010).

[18] Vellekoop, I. M. and Aegerter, C., "Scattered light fluorescence microscopy: imaging through turbid layers," Opt. Lett. 35, 1245-1247 (Apr 2010).

[19] Hsieh, C.-L., Pu, Y., Grange, R., Laporte, G., and Psaltis, D., "Imaging through turbid layers by scanning the phase conjugated second harmonic radiation from a nanoparticle," Opt. Express 18, 20723-20731 (Sep 2010).

[20] Choi, Y., Yang, T. D., Fang-Yen, C., Kang, P., Lee, K. J., Dasari, R. R., Feld, M. S., and Choi, W., "Overcoming the diffraction limit using multiple light scattering in a highly disordered medium," Phys. Rev. Lett. 107, 023902 (Jul 2011).

[21] van Putten, E. G., Akbulut, D., Bertolotti, J., Vos, W. L., Lagendijk, A., and Mosk, A. P., "Scattering lens resolves sub-100 nm structures with visible light," Phys. Rev. Lett. 106, 193905 (May 2011).

[22] Bertolotti, J., van Putten, E. G., Blum, C., Lagendijk, A., Vos, W. L., and Mosk, A. P., "Non-invasive imaging through opaque scattering layers," Nature 491, 232 (2012). 
[23] Yang, X., Pu, Y., and Psaltis, D., "Imaging blood cells through scattering biological tissue using speckle scanning microscopy," Optics Express 22, 3405 (2014).

[24] Katz, O., Heidmann, P., Fink, M., and Gigan, S. Nature Photonics 8, 784 (2014).

[25] Takasaki, K. and Fleisher, J., "Phase-space measurement for depth-resolved memory-effect imaging," Optics Express 22, 31426 (2014).

[26] Goodman, J. W., [Statistical Optics], John Wiley \& Sons (1985).

[27] Feng, S., Kane, C., Lee, P. A., and Stone, A. D., "Correlations and fluctuations of coherent wave transmission through disordered media," Phys. Rev. Lett. 61, 834-837 (Aug 1988).

[28] Akkermans, E. and Montambaux, G., [Mesoscopic Physics of Electrons and Photons], Cambridge University Press (2007).

[29] Freund, I., Rosenbluh, M., and Feng, S., "Memory effects in propagation of optical waves through disordered media," Phys. Rev. Lett. 61, 2328-2331 (Nov 1988).

[30] Anderson, P. W., "Absence of diffusion in certain random lattice," Physical Review 109, 1492 (1958).

[31] Lagendijk, A., van Tiggelen, B., and Wiersma, D. S., "Fifty years of Anderson localization," Physics Today August, 24 (2009).

[32] Fienup, J. R., "Reconstruction of an object from the modulus of its fourier transform," Opt. Lett. 3, 27-29 (Jul 1978).

[33] Fienup, J. R., "Phase retrieval algorithms: a comparison," Appl. Opt. 21, 2758-2769 (Aug 1982).

[34] Dainty, J. C., "Stellar speckle interferometry," in [Laser Speckle and Related Phenomena], Dainty, J., ed., Springer Verlag (1984).

[35] Miao, J., Charalambous, P., Kirz, J., and Sayre, D., "Extending the methodology of x-ray crystallography to allow imaging of micrometre-sized non-crystalline specimens," Nature 400, 342-344 (July 1999).

[36] Abbey, B., Whitehead, L. W., Quiney, H. M., Vine, D. J., Cadenazzi, G. A., Henderson, C. A., Nugent, K. A., Balaur, E., Putkunz, C. T., Peele, A. G., Williams, J., and McNulty, I., "Lensless imaging using broadband x-ray sources," Nat. Photon. 5, 420-424 (July 2011). 